\title{
Spectroscopic Characteristics \\ of the Methanol Decomposition Intermediates \\ on a Platinum Nanocluster
}

\author{
Svetlana S. Laletina*, \\ Aleksey M. Shor, Vladimir A. Nasluzov, \\ Elena A. Ivanova-Shor and Anatoly I. Rubaylo \\ Institute of Chemistry and Chemical Technology SB RAS \\ $F R C$ «Krasnoyarsk Science Center SB RAS» \\ Krasnoyarsk, Russian Federation
}

Received 21.05.2020, received in revised form 01.06.2020, accepted 10.06.2020

\begin{abstract}
The density functional method was used to study vibrational frequenciesof the surface species involved in the reaction of the methanol dehydration via pathway $\mathrm{CH}_{3} \mathrm{OH} \rightarrow \mathrm{CH}_{3} \mathrm{O} \rightarrow \mathrm{CH}_{2} \mathrm{O} \rightarrow \mathrm{CHO} \rightarrow \mathrm{CO}$ a ideal $\mathrm{Pt}(111)$ surface and surface of nanoparticle $\mathrm{Pt}_{79}$. Connection of spectral features with stability and coordination of the adsorbed molecules was discussed. The determined vibrational features enable identification of themain reaction intermediates.
\end{abstract}

Keywords: DFT method, Pt surface and nanoparticles, methanol, dehydrogenation, intermediates, vibrational frequencies.

Citation: Laletina S.S., Shor A.M., Nasluzov V.A., Ivanova-Shor E.A., Rubaylo A.I. Spectroscopic characteristics of the methanol decomposition intermediates on a platinum nanocluster, J. Sib. Fed. Univ. Chem., 2020, 13(2), $273-282$. DOI: 10.17516/1998-2836-0181

(C) Siberian Federal University. All rights reserved

This work is licensed under a Creative Commons Attribution-NonCommercial 4.0 International License (CC BY-NC 4.0).

* Corresponding author E-mail address: shkulepo@rambler.ru 


\title{
Спектроскопические характеристики \\ интермедиатов разложения метанола \\ на нанокластере платины
}

\author{
С.С. Лалетина, А.М. Шор, \\ В.А. Наслузов, Е.А. Иванова-Шор, А.И. Рубайло \\ Институт химии и химической технологии СО РАН \\ ФИЦ «Красноярский научный иентр СО РАН» \\ Российская Федерация, Красноярск
}

\begin{abstract}
Аннотация. Методом функционала плотности рассчитаны частоты колебаний поверхностных комплексов частиц, образующихся в ходе реакции разложения метанола по маршруту $\mathrm{CH}_{3} \mathrm{OH} \rightarrow \mathrm{CH}_{3} \mathrm{O} \rightarrow \mathrm{CH}_{2} \mathrm{O} \rightarrow \mathrm{CHO} \rightarrow \mathrm{CO}$ на идеальной поверхности $\mathrm{Pt}(111)$ и поверхности наночастицы $\mathrm{Pt}_{79}$. Показана связь спектральных характеристик со стабильностью и способом координации частиц на поверхности. Полученные данные позволяют идентифицировать элементарные шаги рассматриваемого реакционного процесса.
\end{abstract}

Ключевые слова: метод функционала плотности, поверхность и наночастицы Рt, метанол, дегидрирование, интермедиаты, частоты колебаний.

Цитирование: Лалетина, С.С. Спектроскопические характеристики интермедиатов разложения метанола на нанокластере платины / С.С. Лалетина, А.М. Шор, В.А. Наслузов, Е.А. Иванова-Шор, А.И. Рубайло // Журн. Сиб. федер. ун-та. Химия, 2020. 13(2). С. 273-282. DOI: 10.17516/1998-2836-0181

\section{Введение}

Изучение гетерогенных каталитических реакций методами колебательной спектроскопии затруднено из-за сложности идентификации промежуточных поверхностных форм, обусловленной их нестабильностью и коротким временем жизни. Дополнительные проблемы при интерпретации колебательных спектров вызывает наличие примесей, а также нерегулярная структура поверхности катализаторов. Значительную помощь в расшифровке спектральных данных могут оказать теоретические методы. В частности, это относится к реакции дегидрирования метанола на платиновых катализаторах - одному из способов получения $\mathrm{H}_{2}$ и $\mathrm{CO}$, проходящей в основном через образование кислородсодержащих частиц $\mathrm{CH}_{\mathrm{x}} \mathrm{O}$ [1]. Разложение и окисление метанола на чистой и обогащенной кислородом поверхности $\mathrm{Pt}(111)$ ранее изучалось методами спектроскопии высокого разрешения характеристических потерь энергии электронов HREELS и температурно-программируемой десорбции TPD [2-4], инфракрасной отражательно-адсорбционной спектроскопии $\operatorname{IRAS}[5,6]$, рентгеновской фотоэлектронной спектроскопии XPS [7], ультрафиолетовой фотоэлектронной спектроскопии, UPS, дифракции медленных электронов $L E E D$ и оже-спектроскопии (AES) [8]. В ходе исследований на регулярной поверхности $\operatorname{Pt}(111)$ зарегистрированы молекулярно адсорбированные $\mathrm{CO}$ и метанол $\left(\mathrm{CH}_{3} \mathrm{OH}\right)$ [4]. Следы метокси$\left(\mathrm{CH}_{3} \mathrm{O}\right)$ и формильных групп (CHO) обнаруживаются только на предварительно окисленной 
поверхности $\mathrm{Pt}(111)-2 \mathrm{x} 2(\mathrm{O})[5,9,10]$. Ранее были выполнены теоретические исследования разложения метанола на поверхности $\operatorname{Pt}(111)$ [11-13]. Были рассчитаны частоты колебаний интермедиатов дегидрирования метанола [14], а также метанола и формальдегида [15].

Известно, что структура поверхности катализатора значительно влияет на его активность. В частности, было показано, что при окислении метанола на дефектной поверхности платины (111) наблюдается более высокая селективность образования СО [7]. Тем не менее прохождение реакции на таких поверхностях ввиду их сложности исследовалось мало $[16,17]$. Для описания дефектной поверхности представляют интерес небольшие наночастицы (НЧ), ограниченные гранями $\{111\}$ и $\{100\}$ [18]. Для учета эффектов наноструктурирования поверхности реакция дегидрирования метанола моделировалась на нанокластерах $\mathrm{Pt}_{79}[19,20]$. Было показано, что адсорбция на вершинах и ребрах нанокластера на 20-70 кДж/моль более предпочтительна, чем на его наногранях $\{111\}$ или на поверхности $\mathrm{Pt}(111)$. Зависимость частот СО колебаний от координации поверхностных атомов $\mathrm{Pt}$ исследовалась на примере кластера $\mathrm{Pt}_{8}$, закрепленного на поверхности $\mathrm{CeO}_{2}$ [21]. Также исследовались спектральные характеристики $\mathrm{CH}_{\mathrm{x}}$ и $\mathrm{CH}_{\mathrm{x}} \mathrm{O}$ частиц, адсорбированных на различных структурных элементах $\mathrm{Pd}_{79}$ [22].

В данной работе мы моделировали колебательные частоты интермедиатов реакции разложения метанола, идущей на регулярной поверхности платины (111) и нанокластере $\mathrm{Pt}_{79}$. Изучено влияние типа поверхности металла на спектральные свойства адсорбированных частиц и оценена возможность их идентификации в экспериментальных условиях.

Модели и детали расчета настоящей работы соответствуют положениям работы [20]. Гармонические частоты колебаний рассчитаны методом конечных разностей, при задании матрицы Гесса использованы смещения атомов в $\pm 1,5$ пм. Энергии адсорбции $\left(\mathbf{E}_{\mathbf{A}}\right)$ рассчитаны с учетом поправок на энергию нулевых колебаний (ZPE), $\Delta \mathrm{E}=1 / 2 \sum_{\mathrm{i}} \mathrm{h}^{*} v_{\mathrm{i}}$.

\section{Результаты расчетов и обсуждение}

Теоретическое изучение реакционного пути $\mathrm{CH}_{3} \mathrm{OH} \rightarrow \mathrm{CH}_{3} \mathrm{O} \rightarrow \mathrm{CH}_{2} \mathrm{O} \rightarrow \mathrm{CHO} \rightarrow \mathrm{CO}$ разложения метанола на идеальной поверхности $\mathrm{Pt}(111)$ и нанокластере $\mathrm{Pt}_{79}$ было выполнено ранее в работе [20]. Геометрии энергетически выгодных изомеров для каждого из реакционных интермедиатов даны на рис. 1. Энергии адсорбции и частоты колебаний данных структур приводятся в табл. 1.

Метанол. В соответствии с экспериментальными данными $[2,6]$ на поверхности $\mathrm{Pt}(111)$ молекула $\mathrm{CH}_{3} \mathrm{OH}$ привязана к tор-центру через атом кислорода (рис. 1a) с энергией в -46 кДж/Моль [23]. Рассчитанная энергия адсорбции равна -31 кДж/моль. При адсорбции метанола на вершине кластера $\mathrm{Pt}_{79}$ (центр top-corner, рис. 1a) энергия $\mathrm{E}_{\mathrm{A}}$ увеличивается (по модулю) до -52 кДж/моль (табл. 1).

Присутствие метанола на поверхности Pt определяется по наличию в спектре полос валентных $\mathrm{OH}$-колебаний. При адсорбции $\mathrm{CH}_{3} \mathrm{OH}$ на вершине кластера и top-центре поверхности $\operatorname{Pt}(111)$ рассчитанные частоты колебаний равны 3694 и 3563 см$^{-1}$ соответственно. В то же время в экспериментах колебания $v(\mathrm{OH})$ наблюдались в интервале частот 3280-3310 см-1 для чистой $[2,4]$ и покрытой кислородом $[2,5]$ поверхности $\mathrm{Pt}(111)$. Столь низкие частоты объясняются участием ОН-групп в водородных связях, возникающих при высоком покрытии поверхности молекулами спирта. При низком покрытии метанолом (0,3 молекулярного слоя $(M C))$ на по-

$$
-275-
$$




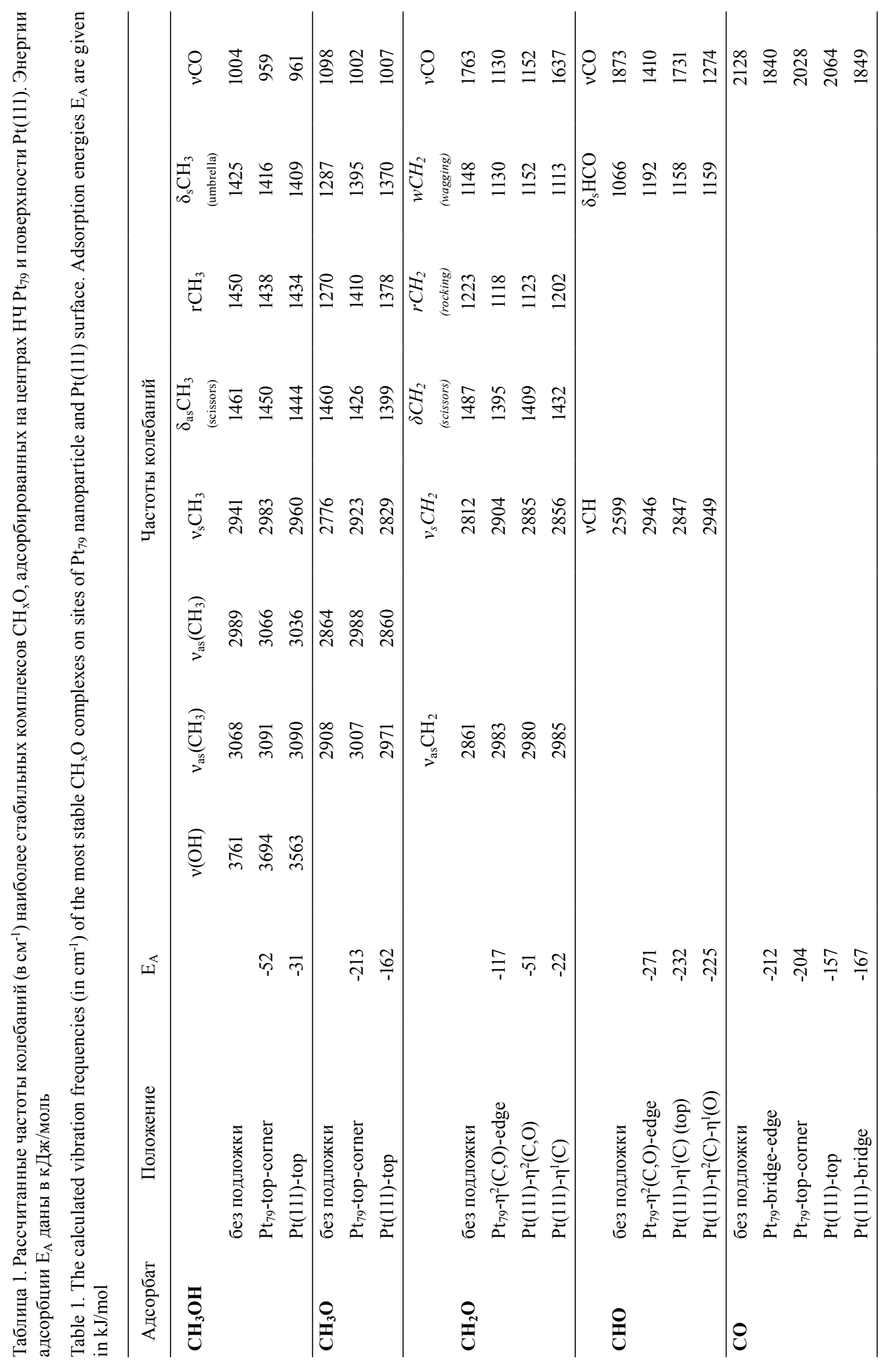



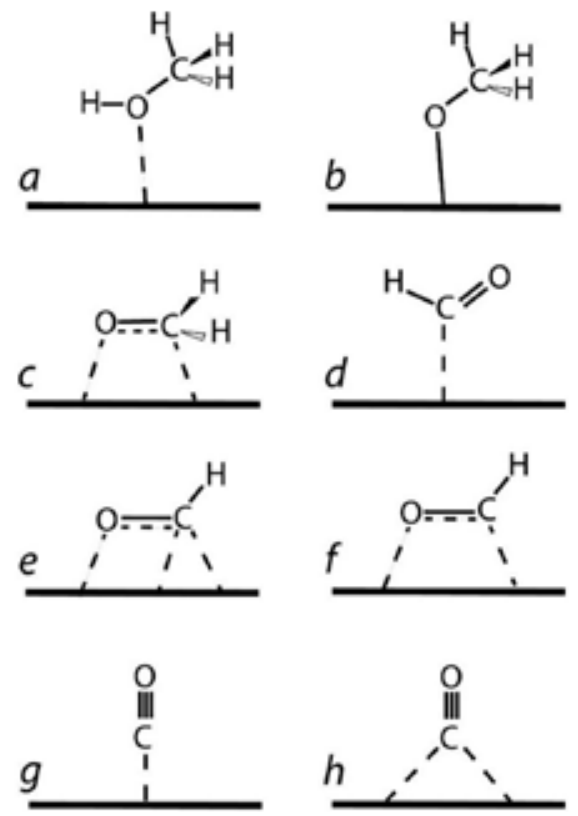

Рис. 1. Способы координации в наиболее стабильных комплексах $\mathrm{CH}_{\mathrm{x}} \mathrm{O}$, найденных в работе [20]: a $\mathrm{CH}_{3} \mathrm{OH}$-top; b - $\mathrm{CH}_{3} \mathrm{O}$-top; c - $\mathrm{CH}_{2} \mathrm{O}-\eta^{2}$; g - CO-top; h - CO-bridge, адсорбированных на поверхностях $\mathrm{Pt}(111)$ и $\mathrm{Pt}_{79} ; \mathrm{d}-\mathrm{CHO}-\mathrm{top}$ и е $-\mathrm{CHO}-\eta^{3}$ на $\mathrm{Pt}(111) ; \mathrm{f}-\mathrm{CHO}-\eta^{2}$ на $\mathrm{Pt}_{79}$

Fig. 1. Coordination modes for the most stable $\mathrm{CH}_{x} \mathrm{O}$ complexesfound in Ref. [20]: a) $\mathrm{CH}_{3} \mathrm{OH}$-top, b) $\mathrm{CH}_{3} \mathrm{O}$-top, c) $\mathrm{CH}_{2} \mathrm{O}-\eta^{2}$, g) CO-top, h) CO-bridge adsorbed on both Pt (111) surface and $\mathrm{Pt}_{79}$; d) $\mathrm{CHO}$-top and e) $\mathrm{CHO}-\eta^{3}$ on $\mathrm{Pt}(111)$; and also f) $\mathrm{CHO}-\eta^{2}$ on $\mathrm{Pt}_{79}$

верхности $\mathrm{Pt}(111)-2 \times 2(\mathrm{O})$ наблюдалась узкая полоса при $3500 \mathrm{~cm}^{-1}$ [5], свидетельствующая о наличии изолированных друг от друга ОН-групп.

Валентные $v\left(\mathrm{CH}_{x}\right)$ - и $v(\mathrm{CO})$-колебания метанола проявляются в областях 2960-3090 и $\sim 960 \mathrm{~cm}^{-1}$ соответственно, и нечувствительны к типу поверхности платины.

Метокси-группа. Согласно расчетам взаимодействие радикальной метокси-частицы с поверхностью $\mathrm{Pt}(111)$ оказывается намного более сильным, чем молекулы $\mathrm{CH}_{3} \mathrm{OH}$. Предпочтительна on-top-адсорбция $\mathrm{CH}_{3} \mathrm{O}$ (рис. 1 ) ), характеризуемая энергией $\mathrm{E}_{\mathrm{A}}=-162$ кДж/моль. Адсорбция на вершине нанокластера (центр top-corner) оказывается более выгодной на 50 кДж/моль (табл. 2). Экспериментально измеренная энергия $\mathrm{E}_{\mathrm{A}}$ для кислородсодержащей $\operatorname{Pt}(111)$ поверхности оценена в $-187 \pm 11$ кДж/моль [24].

Образование поверхностной метокси-группы характеризуется исчезновением из спектра полос, отвечающих $v(\mathrm{OH})$ колебаниям (табл. 1). По сравнению с адсорбированной молекулой метанола рассчитанная длина связи С-О в метокси-группе уменьшается на 4 пм, что отражается в повышении частоты $v(C O)$ на $\sim 40 \mathrm{~cm}^{-1}$ как для $\operatorname{Pt}(111)$, так и для $\mathrm{Pt}_{79}$. Частоты $v\left(\mathrm{CH}_{3}\right)$ колебаний, напротив, понижаются на 120-180 и 60-90 см-1 на $\operatorname{Pt}(111)$ и вершине нанокластера соответственно. Наблюдаемая тенденция хорошо согласуется с экспериментальными данными. Так, например, на предварительно окисленной поверхности $\operatorname{Pt}(111)-2 \mathrm{x} 2(\mathrm{O})$ были зарегистриро-

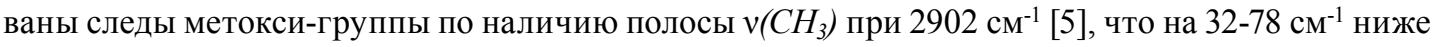
соответствующих частот адсорбированного метанола (2934-2980 см-1).

$$
-277-
$$


Формальдегид. Наиболее прочно $\mathrm{CH}_{2} \mathrm{O}$ адсорбируется при образовании $\eta^{2}(\mathrm{C}, \mathrm{O})$-связи с поверхностью платины (рис. 1c). На поверхности $\mathrm{Pt}(111) \mathrm{E}_{\mathrm{A}}$ равна -56 кДж/моль, в то время как на НЧ $\mathrm{Pt}_{79}$ энергия адсорбции достигает значений -117 кДж/моль. Схожие энергии адсорбции на поверхности $\operatorname{Pt}(111),-55$ и -69 кДж/моль, найдены экспериментально [18]. Стабильность комплекса формальдегида на поверхности $\mathrm{Pt}(111)$ с $\eta^{1}$-координацией атома O составляет всего -22 кДж/моль.

Как показано в работе [15], координация $\mathrm{CH}_{2} \mathrm{O}$ в $\eta^{2}$-положении при прямом взаимодействии атома $\mathrm{C}$ с поверхностью платины приводит к изменению его $\mathrm{sp}^{2}$-гибридизации до частично $\mathrm{sp}^{3}$-состояния и, как следствие, понижению порядка С-О-связи. В результате частота $v(C O)$ понижается с $1763 \mathrm{~cm}^{-1}$ в газовой фазе до $1152 \mathrm{~cm}^{-1}$ (на $\left.\operatorname{Pt}(111)\right)$ и 1130 (на $\left.\mathrm{Pt}_{79}\right) \mathrm{cm}^{-1}$. Показательно, что в случае $\eta^{1}$-координации частота понижается только до $1637 \mathrm{~cm}^{-1}$. Последняя величина хорошо соотносится с экспериментальной частотой, измеренной на предварительно окисленной поверхности $\mathrm{Pt}(111)-2 \times 2(0)$ [5].

Частоты валентных $v\left(\mathrm{CH}_{2}\right)$ колебаний наблюдаются в той же спектральной области, что и в случае поверхностной метокси-группы, 2856-2983 см-1, однако число полос уменьшается до двух.

Формил. В соответствии с данными предыдущих расчетов $[11,12]$ для частицы СНО на поверхности $\mathrm{Pt}(111)$ были найдены два близких по энергии способа адсорбции. В первом случае ( $\eta^{1}$-координация) формил связан с top-центром поверхности через атом С (рис. $1 d$ ), во втором адсорбция происходит через бидентатно связанный атом $\mathrm{C}\left(\eta^{2}\right)$ и атом $\mathrm{O}\left(\eta^{1}\right)$ (рис. 1e). Соответствующие энергии адсорбции равны -232 и -225 кДж/моль. На $\mathrm{Pt}_{79}$ частица СНО координируется бидентатно на ребре нанокластера (рис. $1 f$ ) с энергией $\mathrm{E}_{\mathrm{A}}=-271$ кДж/моль.

Формильная группа, адсорбированная в $\eta^{1}(\mathrm{C})$-положении, характеризуется частотой

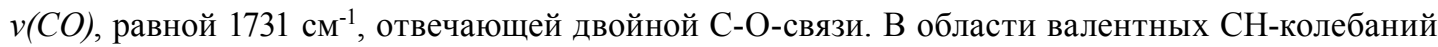
присутствует единственная полоса с частотой $v(\mathrm{CH})$, равной $2847 \mathrm{~cm}^{-1}$. Мостиковые двух- и трехкоординированные формы $\mathrm{CHO}$, как и в случае $\mathrm{CH}_{2} \mathrm{O}$, демонстрируют понижение порядка С-О-связи с соответствующим понижением частоты $v(C O)$ до $1410 \mathrm{~cm}^{-1}$ на нанокластере $\mathrm{Pt}_{79}$ и $1274 \mathrm{~cm}^{-1}$ на поверхности $\operatorname{Pt}(111)$. В то же время рассчитанные частоты $v(C H)$ равны 2949 и $2946 \mathrm{~cm}^{-1}$ соответственно.

Экспериментально удалось зафиксировать $v(C O)$ полосу в диапазоне частот 1600-1700 cм-1, отнесенную к $\eta^{1}$-комплексу СНО на окисленной поверхности платины $[9,10,25]$.

Моноокси углерода. Нами рассмотрены терминальная (top-) (рис. 1 ) и и мостиковая (bridge-) (рис. 1h) адсорбция оксида углерода на поверхности платины. Стабильности top- и bridge-поверхностных форм СО отличаются не более чем на 10 кДж/моль (табл. 1) и могут рассматриваться как приблизительно одинаковые. В то же время на $\mathrm{HЧ} \mathrm{Pt}_{79}$ энергия адсорбции СО на 50 кДж/моль выше, чем на $\mathrm{Pt}(111)$. Это связано с тем, что на $\mathrm{Pt}_{79}$ молекула СО предпочтительно адсорбируется на ребрах (центр bridge-edge) и вершинах (центр top-corner) нанокластера. На гранях нанокластера энергия адсорбции СО заметно меньше и приближается к таковой, рассчитанной на поверхности $\operatorname{Pt}(111)[20,26]$.

Структурные и, как следствие, спектральные свойства адсорбированной молекулы СО описываются в рамках модели переноса заряда между $\mathrm{CO}$ и атом металла: $\mathrm{CO}(\sigma) \rightarrow \mathrm{M}\left(\sigma^{*}\right)$ (донирование) и $\mathrm{M}(\pi) \rightarrow \mathrm{CO}\left(\pi^{*}\right)$ (обратное донирование) [27], при этом второй эффект играет основ- 
ную роль в увеличении длины С-О-связи и понижении частоты $v(C O)$. Чем с большим числом атомов металла взаимодействует молекула $\mathrm{CO}$, тем ниже частота $v(C O)$. В случае монодентатной top-координации рассчитанные частоты $v(C O)$ на $\mathrm{Pt}_{79}$ и поверхности $\mathrm{Pt}(111)$ равны 2028 и $2064 \mathrm{~cm}^{-1}$ соответственно. Мостиковая координация понижает частоты до 1840 и $1849 \mathrm{~cm}^{-1}$.

Найденная в расчетах тенденция согласуется с данными эксперимента. Для topкоординации наблюдаемые частоты $v(C O)$ равны 2081-2100 см$^{-1}$ [28]. Мостиковая адсорбция ведет к понижению частоты до $1850 \mathrm{~cm}^{-1}$ [28].

\section{Заключение}

Расчетные данные о колебательных характеристиках реагентов, интермедиатов и продуктов реакции разложения метанола на поверхности платины, собранные в табл. 1, позволяют прийти к вполне определенным заключениям о возможности связи изменений, происходящих в колебательных спектрах в ходе реакции, с образованием тех или иных поверхностных форм.

При этом необходимо отметить, что мы не рассматривали колебания адсорбированных молекул относительно поверхности адсорбента (не приведены в табл. 1). Полосы таких колебаний располагаются в нижней области спектра (обычно ниже $600 \mathrm{~cm}^{-1}$ ) и являются малоинформативными. Спектральная область вплоть до $1500 \mathrm{~cm}^{-1}$ в основном содержит полосы деформационных колебаний $-\mathrm{CH}_{\mathrm{x}}(\mathrm{x}=1-3)$ и $-\mathrm{CHO}$ групп. Характерная особенность данной области спектра, проявляющаяся в ходе реакции, - это уменьшение числа полос колебаний в ряду $\mathrm{CH}_{3} \mathrm{OH}>\mathrm{CH}_{3} \mathrm{O}>\mathrm{CH}_{2} \mathrm{O}>\mathrm{CHO}$ как результат последовательного отрыва атомов водорода. Наиболее простая картина в данной области спектра наблюдается в случае формильных поверхностных форм. В спектре адсорбированного формила наблюдаются полосы в относительно узком диапазоне частот 1150-1200 cм-1, отнесенные к деформационному колебанию $\delta(H C O)$. Отметим, что положение полосы данного колебания хорошо коррелирует с энергией адсорбции частицы СНО на разных типах платинового субстрата, но является нечувствительным к способу координации формила к поверхности $\mathrm{Pt}(111)$ (табл. 1).

Обращаясь к валентным колебаниям адсорбированных метанола, $\mathrm{CO}$ и рассмотренных в данной работе интермедиатов, необходимо указать на колебание СО-группы. Валентное СОколебание является наиболее информативной характеристикой, с помощью которой возможно однозначно определить образование каждой из изученных форм. При последовательном отщеплении атомов Н полоса $v(C O)$ смещается в область высоких частот - от 959 до $2064 \mathrm{~cm}^{-1}$. Это связано с изменением порядка СО-связи в адсорбированных частицах - от 1 в метаноле до 3 в оксиде углерода. В случае частиц СНО и $\mathrm{CO}$ высокая чувствительность полосы $v(C O)$ позволяет различить формы с разным способом координации к поверхности платины. К сожалению, в случае адсорбированных частиц $\mathrm{CH}_{3} \mathrm{OH}, \mathrm{CH}_{3} \mathrm{O}$ и $\mathrm{CH}_{2} \mathrm{O}$ полосы валентного $\mathrm{CO}$ колебания идеформационных $-\mathrm{CH}_{\text {x }}$ колебаний перекрываются, что в экспериментальных условиях может затруднить определение частоты $v(C O)$.

Спектральная область выше $2800 \mathrm{~cm}^{-1}$ соответствует частотам валентных колебаний $-\mathrm{CH}_{\mathrm{x}}-$ и -OH-групп. При этом полосы $v\left(\mathrm{CH}_{x}\right)$ наблюдаются в области ниже $3100 \mathrm{~cm}^{-1}$, тогда как $v(\mathrm{OH})$ проявляют себя выше $3500 \mathrm{~cm}^{-1}$, т.е. обе группы полос хорошо разделены. Наличие в колебательном спектре полос $v(\mathrm{OH})$ позволяет однозначно определить присутствие на поверхности $\mathrm{Pt}$ адсорбированного $\mathrm{CH}_{3} \mathrm{OH}$. Более того, валентное колебание гидроксильной группы метанола 
демонстрирует чувствительность к координационному окружению атома Pt. Адсорбция спирта на атоме металла, находящемся в более высоком координационном окружении, приводит к большему понижению частоты $v(\mathrm{OH})$ относительно рассчитанной в газовой фазе. Исчезновение полос $v(\mathrm{OH})$ из спектра свидетельствует о разрыве О-Н-связи и является одним из доказательств прохождения реакции по рассматриваемому в данной работе реакционному пути. В ряду превращений $\mathrm{CH}_{3} \mathrm{OH} \rightarrow \mathrm{CH}_{3} \mathrm{O} \rightarrow \mathrm{CH}_{2} \mathrm{O} \rightarrow \mathrm{CHO}$ наблюдается тенденция к понижению частот полос $v\left(\mathrm{CH}_{x}\right)$ от 3091 до $2847 \mathrm{~cm}^{-1}$ (верхняя граница рассчитанного диапазона). Помимо этого, последовательный отрыв атомов Н от атома углерода должен приводить к уменьшению числа полос $v\left(C H_{x}\right)$ колебаний. В пределе, в случае адсорбированного формила, должна наблюдаться одна полоса $v(\mathrm{CH})$. Однако необходимо иметь в виду, что адсорбция на металлических центрах различной природы (идеальная поверхность, дефекты), различные способы связи с поверхностью могут приводить к усложнению наблюдаемого спектра.

Подводя итог вышесказанному, можно заключить, что спектральные характеристики поверхностных комплексов, образующихся в ходе разложения метанола, позволяют однозначно идентифицировать образование адсорбированных форм метанола (присутствие полосы $v(O H)$ ), оксида углерода (наличие единственной полосы $v(C O)$ ), а также формильных групп. В последнем случае колебательный спектр представляет собой относительно простой набор хорошо

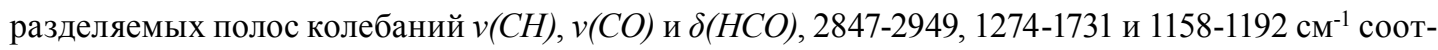
ветственно. Фиксация поверхностных $\mathrm{CH}_{3} \mathrm{O}$ и $\mathrm{CH}_{2} \mathrm{O}$ форм наиболее проблематична. Основным спектральным критерием их образования может служить изменение в положении полос $v\left(\mathrm{CH}_{x}\right)$.

\section{Благодарности / Acknowledgements}

Работа выполнена в рамках государственного задания Института химии и химической технологии СО РАН (проект АААА-А17-117021310221-7). Авторы благодарят Сибирский суперкомпьютерный центр (г. Новосибирск) и суперкомпьютерный комплекс МГУ имени М.В. Ломоносова [29] за предоставленные вычислительные ресурсы.

The work was performed within the framework of the state project of the Institute of Chemistry and Chemical Technology of SB RAS (project AAAA-A17-117021310221-7). The authors are grateful to the Siberian Supercomputer Center (Novosibirsk) and the supercomputer complex of Lomonosov Moscow State University [29] for the provided computing resources.

\section{Список литературы / References}

1. Davis J.L., Barteau M.A. Decarbonylation and decomposition pathways of alcohol's on Pd(111). Surf. Sci. 1987. Vol. 187, P. 387-406

2. Sexton B.A. Methanol decomposition on platinum (111). Surf. Sci. 1981. Vol. 102, P. 271-281.

3. Henderson M.A., Mitchell G.E., White J.M. The decomposition of formaldehyde on Pt(111): A TPD and HREELS study. Surf. Sci. 1987. Vol. 188, P. 206-218.

4. Skoplyak O., Menning C.A., Barteau M.A., Chen J.G. Experimental and theoretical study of reactivity trends for methanol on CoPt (111) and NiPt (111) bimetallic surfaces. J. Chem. Phys. 2007. Vol. 127, P. 114707-10.

5. Liu Z., Sawada T., Takagi N., Watanabe K., Matsumoto Y. Reaction intermediates in the oxidation of methanol on a Pt(111)-(2×2)O surface. J. Chem. Phys. 2003. Vol. 119, P. 4879-4886. 
6. Ehlers D.H., Spitzer A., Lüth H. The adsorption of methanol on $\operatorname{Pt}(111)$, an IR reflection and UV photoemission study. Surf. Sci. 1985. Vol. 160, P. 57-69.

7. Kaichev V.V., Prosvirin I.P., Bukhtiyarov V.I. Decomposition and oxidation of methanol on platinum: A study by in situ X-ray photoelectron spectroscopy and mass spectrometry. Kinet. Catal. 2014. Vol. 55, P. 505-519.

8. Abbas N.M., Madix R.J. The effects of structured overlayers of sulfur on the kinetics and mechanism of simple reactions on Pt(111): I. Formaldehyde decomposition. Appl. Surf. Sci. 1981. Vol. 7, P. 241-275.

9. Zhang Y., Weaver M.J. Application of surface-enhanced Raman spectroscopy to organic electrocatalytic systems: decomposition and electrooxidation of methanol and formic acid on gold and platinum-film electrodes. Langmuir 1993. Vol. 9, P. 1397-1403.

10. Beden B., Juanto S., Leger J.M., Lamy C. Infrared spectroscopic study of the methanol adsorbates at a platinum electrode: Part III. Structural effects and behaviour of a polycrystalline surface. J. Electroanal. Chem. 1987. Vol. 238, P. 323-331.

11.Greeley J., Mavrikakis M. A First-Principles Study of Methanol Decomposition on $\operatorname{Pt}(111) . J$. Am. Chem. Soc. 2002. Vol. 126, P. 7193-7201.

12. Desai S.K., Neurock M., Kourtakis K. A periodic density functional theory study of the dehydrogenation of methanol over Pt(111). J. Phys. Chem. B 2002. Vol. 106, P. 2559-2568.

13. García-Muelas R., Li Q., López N. Density functional theory comparison of methanol decomposition and reverse reactions on metal surfaces. ACS Catal. 2015. Vol. 5, P. 1027-1036.

14. Kandoi S., Greeley J., Sanchez-Castillo M., Evans S.T., Gokhale A., Dumesic J., Mavrikakis M. Prediction of experimental methanol decomposition rates on platinum from first principles. Top. Catal. 2006. Vol. 37, P. 17-27.

15. Bakó I., Pálinkás G. Vibration of small molecules on Pt(111) surface. Surf. Sci. 2006. Vol. 600, P. 3809-3814.

16. Niu C.-Y., Jiao J., Xing B., Wang G.-C., Bu X.-H. Reaction mechanism of methanol decomposition on Pt-based model catalysts: a theoretical study. J. Comput. Chem. 2010. Vol. 31, P. 2023-2037.

17. Tomaschun G., Klüner T. Methanol oxidation on the $\operatorname{Pt}(321)$ surface: a theoretical approach on the role of surface morphology and surface coverage effects. Phys. Chem. Chem. Phys. 2019. Vol. 21, P. $18227-18239$.

18. Barmparis G.D., Lodziana Z., Lopez N., Remediakis I.N. Nanoparticle shapes by using Wulff constructions and first-principles calculations. Beilstein J. Nanotechnol. 2015. Vol. 6, P. 361-368.

19. Mahata A., Choudhuri I., Pathak B. A cuboctahedral platinum $\left(\mathrm{Pt}_{79}\right)$ nanocluster enclosed by well-defined facets favours di-sigma adsorption and improves the reaction kinetics for methanol fuel cells. Nanoscale 2015. Vol. 7, P. 13438-13451.

20. Laletina S.S., Shor E.A., Mamatkulov M.I., Yudanov I.V., Kaichev V.V., Bukhtiyarov V.I. Theoretical Study of the Methanol Dehydrogenation on Platinum Nanocluster. J. SFU. Chem. 2016. Vol. 9(4), P. 430-442. (In Russ.)

21. Aleksandrov H.A., Neyman K.M., Hadjiivanov K.I., Vayssilov G.N. Can the state of platinum species be unambiguously determined by the stretching frequency of an adsorbed $\mathrm{CO}$ probe molecule? Phys. Chem. Chem. Phys. 2016. Vol. 18, P. 22108-22121. 
22. Kozlov S.M., Cabeza G.F., Neyman, K.M. Bonding and vibrations of $\mathrm{CH}_{\mathrm{x}} \mathrm{O}$ and $\mathrm{CH}_{\mathrm{x}}$ species $(\mathrm{x}=1-3)$ on a palladium nanoparticle representing model catalysts. Chem. Phys. Lett. 2011. Vol. 506, P. 92-97.

23. Villegas I., Weaver M.J. Infrared spectroscopy of model electrochemical interfaces in ultrahigh vacuum: Surface-cation solvation in the $\mathrm{Pt}(111) / \mathrm{K}+-$ methanol system. J. Chem. Phys. 1995. Vol. 103, P. 2295-2307.

24. Karp E.M., Silbaugh T.L., Crowe M.C., Campbell C.T. Energetics of adsorbed methanol and methoxy on Pt(111) by microcalorimetry. J. Am. Chem. Soc. 2012. Vol. 134, P. 20388-20395.

25. Lopes M.I., Fonseca I., Olivi P., Beden B., Hahn F., Léger J.M., Lamy C. Integrated electromodulated IR reflectance spectroscopy bands. Part 2: Methanol adsorbates at polycrystalline platinum and $\mathrm{Pt}(111)$ single-crystal electrodes in acid medium. J. Electroanal. Chem. 1993. Vol. 346, P. $415-432$.

26. Laletina S.S., Mamatkulov M., Shor E.A., Kaichev V.V., Genest A., Yudanov I.V., Rösch N. Size-Dependence of the adsorption energy of $\mathrm{CO}$ on Pt nanoparticles: Tracing two intersecting trends by DFT calculations. J. Phys. Chem. C 2017. Vol. 121, P. 17371-17377.

27. (a) Dewar M.J.S. A Review of the $\pi$-Complex Theory. Bull. Soc. Chim. Fr. 1951. Vol. 18, P. 71-79; (б) Chatt J., Duncanson L.A. Olefin co-ordination compounds. Part III. Infra-red spectra and structure: attempted preparation of acetylene complexes. J. Chem. Soc. 1953. P. 2939-2947.

28. Steininger H., Lehwald S., Ibachi H. On the adsorption of CO on Pt(111). Surf. Sci. Lett. 1982. Vol. 123, P. 264-282.

29. Sadovnichy V., Tikhonravov A., Voevodin V., Opanasenko V. “Lomonosov”: Supercomputing at Moscow State University. In Contemporary High Performance Computing: From Petascale toward Exascale (Chapman \& Hall/CRC Computational Science). USA, Boca Raton: CRC Press, 2013. 283$307 \mathrm{p}$. 